Bull. Mater. Sci, Vol, 1, No. 2, October 1979, pp. 121-128, (C) Printed in India.

\title{
Measurement of magnetic properties of single crystal YIG by non-resonant method
}

\author{
D N BOSE*, S R BORGAONKAR and T S VEDAVATHY \\ Department of Electrical Communication Engincering, Indian Institute of Science, \\ Bangalore 560012 \\ * Present address: Materials Science Centre, Indian Institute of Technology, \\ Kharagpur 721302.
}

MS received 11 August 1979

\begin{abstract}
A non-resonant microwave technique has been employed for the determination of saturation magnetisation $\left(4 \pi M_{8}\right)$ and $g$-factor of pure and Gd-doped single crystal YIG spheres yielding accurate values. It was found that $4 \pi M_{8}=$ $1720-1751 \mathrm{~g}$ for undoped and $1595 \mathrm{~g}$ for $10 \%$ Gd-doped YIG spheres while $g_{\text {eff }}=$ $2 \cdot 00-2 \cdot 0039$. The advantages of this method are simplicity and complete absence of propagation corrections necessary in cavity resonance experiments.
\end{abstract}

\section{Introduction}

The important parameters of single crystal yttrium iron garnet $\left(\mathrm{Y}_{3} \mathrm{Fe}_{5} \mathrm{O}_{12}\right.$ or $\left.\mathrm{YIG}\right)$ spheres are (i) the saturation magnetisation $4 \pi M_{s}$, (ii) the $g$-factor $g_{\text {eff }}$, (iii) linewidth $\triangle H$ and anisotropy field $K_{1} / M_{s}$. Rapid and accurate measurement of these parameters is often required for characterisation of spheres required for applications in tunable devices such as filters and oscillators in the microwave frequency range. The manner in which important device characteristics are determined by these parameters has been reviewed in detail (Matthaei et al 1964). This paper describes the measurement of these parameters by a simple non-resonant waveguide technique and discusses the results obtained in comparison with other established techniques. The main advantage is the absence of propagation corrections which must be used in all cavity methods for accurate determination of $4 \pi M_{s}$ and $g_{\text {eff }}$.

\section{Measurement of $4 \pi M_{s}$}

Measurement of saturation magnetisation $4 \pi M_{s}$ is carried out by (i) ballistic methods, (ii) methods using vibrating coil or vibrating sample magnetometer, (iii) force methods and (iv) microwave methods. The microwave methods consist of determination of (a) demagnetisation effect of sample on resonance (Narayan et al 1969), (b) cavity absorption characteristics (Artmann and Tannenwald 1955), (c) cut-off frequency (Helsajn 1972) and (d) frequencies of higherorder magnetostatic modes (Fletcher et al 1959; Lam 1965; Magid 1964). 
For narrow line-width material such as single crystal YIG the lact method is convenient because the same experimental arrangement can be used for measuring line-width and magnetic anisotropy. Also the measured quantities, which are the differences between magnetic field values can be accurately determined by using a proton resonance magnetometer.

\section{Theory}

$\mathrm{Y}_{3} \mathrm{Fe}_{5} \mathrm{O}_{12}$ has a cubic garnet structure $(a=12 \cdot 376 \AA)$ with 3 types of cation sites. The 3 non-magnetic $\mathrm{Y}^{3+}$ ions occupy $c$ sites which have dodecahedral co-ordination, $2 \mathrm{Fe}^{3+}$ ions occupy octahedral $a$ sites and the remaining $3 \mathrm{Fe}^{3+}$ jons are in tetrahedral $d$ sites. Thus the net magnetisation is due to one $\mathrm{Fe}^{3+}$ ion which has a spin magnetic moment of $5 \mu_{B}$. There are 8 formula units per unit cell and the saturation magnetisation is $4 \pi M_{s}=2460 \mathrm{~g}\left(0^{\circ} \mathrm{K}\right)$, in good agreement with theory, decreasing to $1750 \mathrm{~g}$ at $300^{\circ} \mathrm{K}$. $\mathrm{Gd}^{3+}$ enters the lattice in the $c$ sites with its spin magnetic moment $7 \mu_{B}$ aligned anti-parallel to that in the $d$ sites thus decreasing $4 \pi M_{s}$ at low concentrations.

When a YIG sphere is placed in a static magnetic field $H_{0}$, the application of an orthogonal rf magnetic field causes the spins to precess at a frequency

$$
f_{0}=\gamma\left(H_{0}+k H_{a}\right) \simeq \gamma H_{0},
$$

where $\gamma=$ gyromagnetic ratio $2 \cdot 8 \mathrm{MHz} / \mathrm{Oe}, k=$ constant dependent on sphere orientation and $H_{a}=$ anisotropy field. Due to inhomogeneities in the static and rf magnetic fields or relatively large sphere diameter, higher-order magnetostatic modes are also generated whose frequencies differ from $H_{0}$ by amounts determined. by $4 \pi M_{8}$.

Fletcher and Bell (1959) developed a general theory to predict the resonant frequency of any mode for a sphere at given $H_{0}$ which gives

$$
f_{n m r}=\gamma \cdot 4 \pi M_{s}\left(\Omega_{i}+F_{n m_{r}}\right) \text {, }
$$

where $F_{n m r}$, the form-factor of each mode, has been calculated theoretically and given in table 1 . The index $(n m r)$ describes each magnetostatic mode, where $n$ describes the periodicity of the magnetic potential in $\theta$, the polar angle; $m$ describes the periodicity in the co-ordinate $\phi$ and $r$ is the order of the root of the transcendental equation which is to be satisfied by the boundary condition. In (2)

$$
\Omega_{\mathrm{i}}=H_{\mathrm{i}} / 4 \pi M_{\mathrm{s}}=\left(H_{0}-N_{t} .4 \pi M_{\mathrm{s}}\right) / 4 \pi M_{s} \text {. }
$$

For a sphere the demagnetising factor $N_{t}=1 / 3$ and hence

$$
f_{n m r}=\gamma\left[H_{0}-4 \pi M_{s}\left(1 / 3-F_{n m r}\right)\right] \text {. }
$$

Table 1. Form factor of magnetostatic modes.

\begin{tabular}{lcccccccc}
\hline Mode & 210 & 320 & 110 & 540 & 220 & 330 & 440 & 550 \\
\hline$F_{n m r}$ & 0.20 & 0.286 & 0.333 & 0.363 & 0.40 & 0.420 & 0.445 & 0.455 \\
\hline
\end{tabular}


Table 1 gives $F_{n m}$ for modes with $n-m=0$ or 1 and $r=0$. It is only for these modes that $F_{u m r}$ is independent of $\Omega_{i}$ and hence these modes tune linearly with $H_{0}$. The uniform precession mode is the (110) mode for which $F_{n m_{r}}=1 / 3$ and hence $f_{n m_{r}} \simeq \gamma H_{0}$, if $H_{a}$ is neglected for the moment since $H_{a} \ll H_{0}$.

For a fixed measurement frequency $f$, the static field $H_{n m s}$ required for $(n m r)$ mode is given in terms of the field required for the (110) mode $H_{110}$ by

$$
H_{n m r}-H_{110}=-\left(F_{n m r}-F_{110}\right) 4 \pi M_{s},
$$

and hence

$$
4 \pi M_{s}=\left(H_{n m_{r}}-H_{110}\right) /\left(F_{110}-F_{n m_{r}}\right) .
$$

Hence by accurately measuring the magnetic field difference given by the numerator and identifying the (nmr) mode and thus knowing $F_{n m r}, 4 \pi M_{s}$ can be calculated. Equation (4) can be re-written as

$$
F_{n m \xi}=1 / 3+\frac{\left(f_{n m r} / \gamma\right)-H_{0}}{4 \pi M_{8}}
$$

A plot of the rhs of equation (7) $v s H_{0} / 4 \pi M_{s}$ for different modes given by Fletcher et al (1959) is shown in figure 1. It is seen that for modes for which $n-m=0$

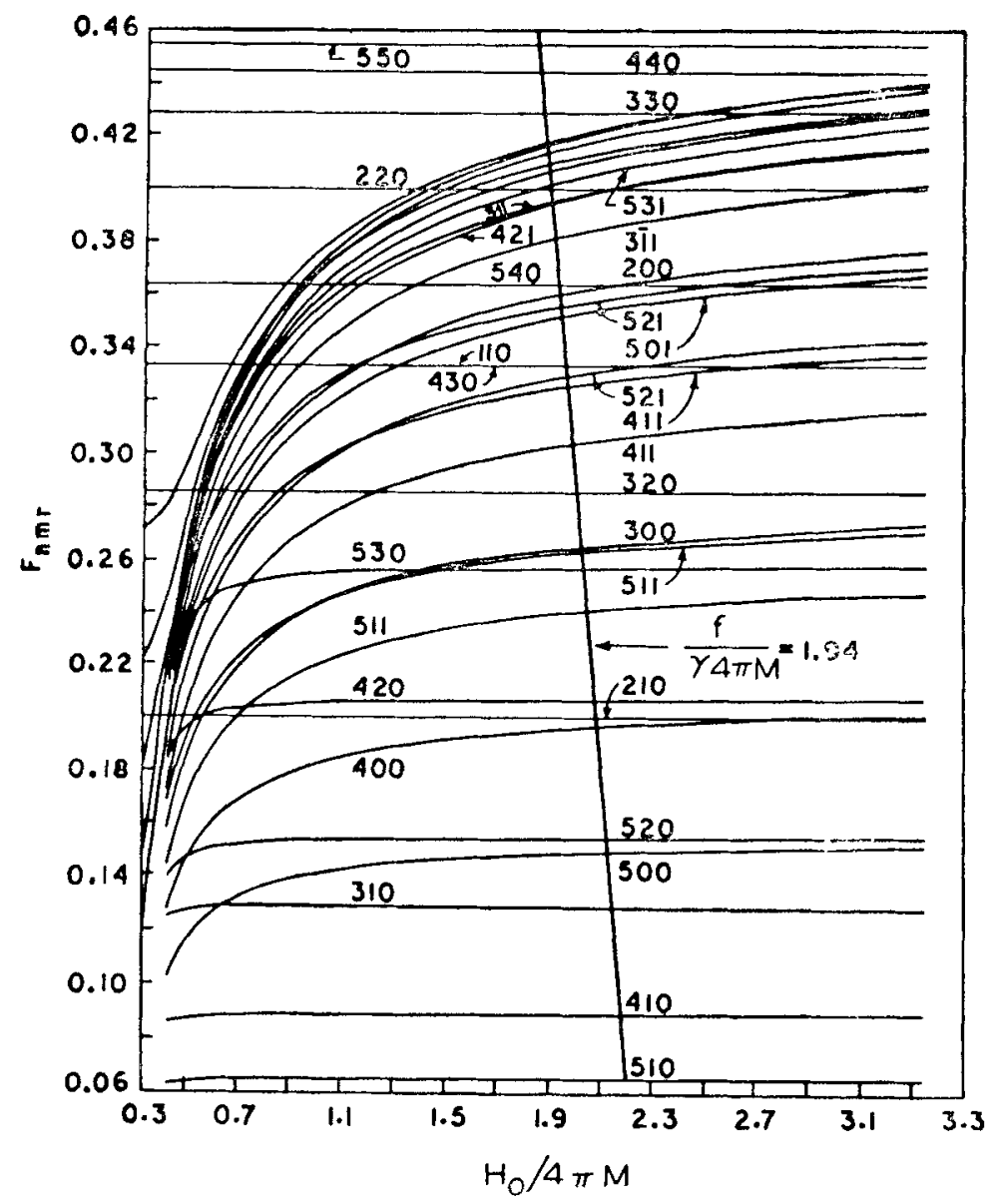

Figure 1. Mode-chart of magnetostatic modes giving form-factor $F_{n m}$, vs. $H_{0} / 4 \pi M$ for modes with $n$ upto 5 . The straight line represents a frequency $f=9 \cdot 709 \mathrm{GHz}$. 
or 1 and $r=0, F_{n m r}$ is constant and $f$ varies linearly with $H_{0}$. These modes are used for the determination of $4 \pi M_{g}$. Intersecting the mode curves is a straight line with $f /(\gamma .4 \pi M)=1.94$ corresponding to a fixed frequency $f=9 \cdot 709 \mathrm{GHz}$. The figure thus indicates that as the static field $H_{0}$ is increased the modes excited successively are (550), (440), (330), (220), (540), (110), (320), (210), etc.

The uniform precession or (110) mode has the highest intensity. The relative intensities of the modes have been tabulated by Fletcher et al (1959) and are in the order $(110)>(540)>(220)>(330)>(320)>(210)$. This provides a guide to the identification of modes although the field $H_{0}$ at which the modes are excited is a more precise identification factor.

In the first method of determining $4 \pi M_{8}$ (table 2) eq. (6) has been used in identifying the (110) and (nmr) modes and measuring the magnetic field separation $H_{n m r}-H_{110}$. It was however found that in some cases mode identification was not unambiguous leading to a large spread in the values of $4 \pi M_{s}$, e.g., for the sphere IGD - 1, the values were $1559 \mathrm{~g}, 1436 \mathrm{~g}$ and $1438 \mathrm{~g}$.

A new method of mode identification was thus evolved using the phenomenon of mode-crossing. Figure 1 shows that the linearly tuning or flat (200) mode intersects the non-flat (540) when $F_{540}=F_{200}=0.364$. From the $x$-axis of the mode-chart at this frequency $H_{540} / 4 \pi M_{s}=H_{200} / 4 \pi M_{s}=2 \cdot 18$. Substituting in (7),

$$
f_{0} /\left(\gamma \cdot 4 \pi M_{s}\right)=2 \cdot 211
$$

where $f_{c}=$ mode intersection frequency. The iterative method to find $4 \pi M_{8}$ is as follows. A value of $4 \pi M_{s}$ is assumed. Using (8) or equivalent expression for the expected mode, $f_{c}$ is computed.

The static field is adjusted to give $f_{0}=f_{n m r}$ and $f_{n m r}-f_{110}$ is measured. With the assumed value of $4 \pi M_{s}, R=\left(f_{n m_{r}}-f_{110}\right) / \gamma \cdot 4 \pi M_{s}$ is calculated. In the example

Table 2. $4 \pi M_{s}$ determination from magnetostatic modes.

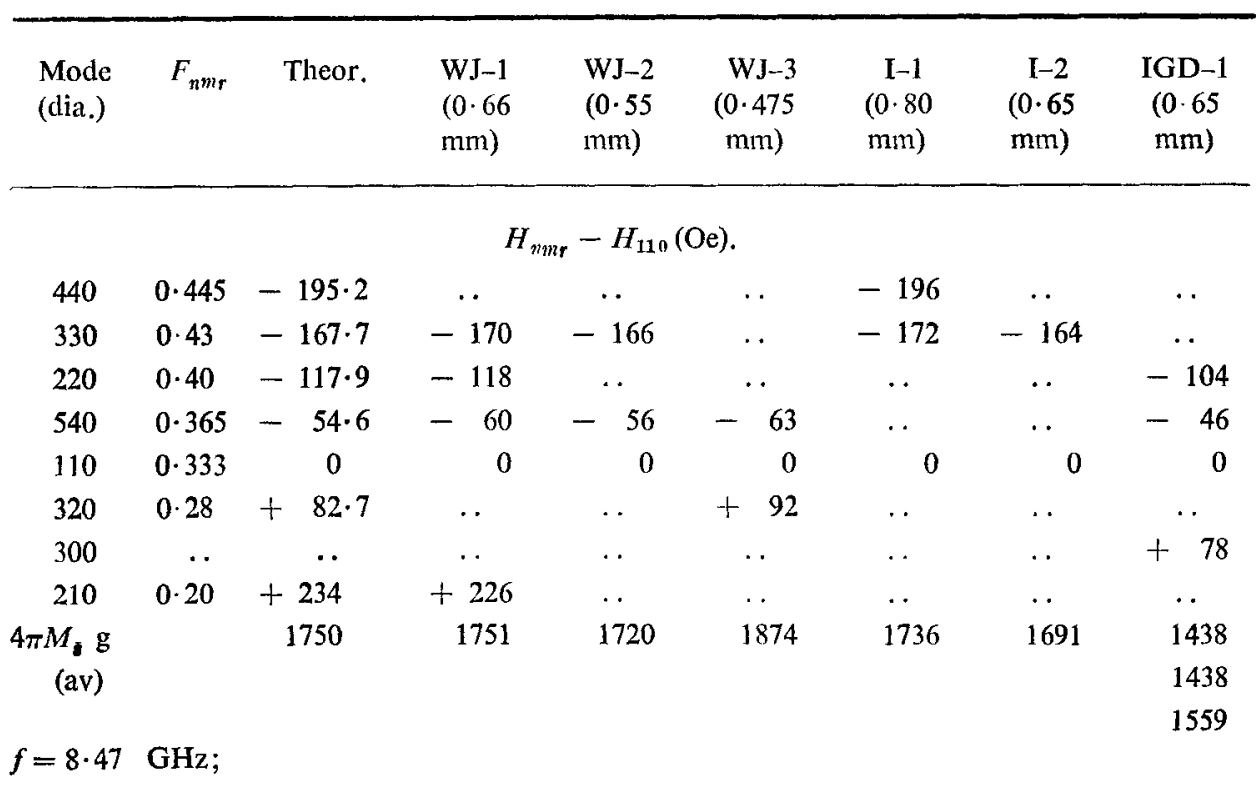


Table 3. $4 \pi M_{s}$ using mode-crossing technique.

\begin{tabular}{ccccc}
\hline Step No. & $\begin{array}{c}\text { Assumed } \\
4 \pi M_{s}(\mathrm{~g})\end{array}$ & $f_{\mathrm{o}}(\mathrm{GHz})$ & $\begin{array}{c}f_{x}-f_{\mathrm{fl0}} \\
(\mathrm{MHz}) \operatorname{expt.}\end{array}$ & $R$ \\
\hline 1. & 1635 & 10.1 & 134.39 & 0.0290 \\
2. & 1735 & 10.7 & 145.5 & 0.0299 \\
3. & 1835 & $11 \cdot 35$ & 164.2 & 0.0319 \\
4. & 1785 & 11.04 & 156.79 & 0.0313 \\
5. & 1760 & 10.89 & 153.06 & 0.0310 \\
\hline
\end{tabular}

given if $R$ is found to be $>0.031$, the assumed value of $4 \pi M_{8}$ is more than the actual value. Depending on whether or not $R>0.031$ a new value of $4 \pi M_{s}$ is assumed and the procedure repeated till $R=0.031$. The simple bisection algorithm is used to modify the $4 \pi M_{s}$ values in successive iterations and gives the correct value. The results are given in table 3 .

\section{Measurement of $g_{\text {eff }}$}

In the 2-sublattice model, the effective $g$-factor is given by

$$
g_{\text {cff }}=g_{A} g_{B}\left(M_{A}-M_{B}\right) /\left(g_{B} M_{A}-g_{A} M_{B}\right),
$$

for the case of negligible damping. In pure YIG the only magnetic ion presents is $\mathrm{Fe}^{3+}$ which has a ground state ${ }^{6} \mathrm{~S}_{5 / 2}(L=0)$ and is distributed in the $a$ and $d$ sites for each of which it is expected that $g_{A}=g_{B}=2 \cdot 00$, giving $g_{\text {cff }}=2 \cdot 00$. The experimental values of $g_{\text {epp }}$ given by von Aulock $(1965)$ are: $2 \cdot 0025\left(150^{\circ} \mathrm{K}\right)$, $2 \cdot 005\left(300^{\circ} \mathrm{K}\right)$ and $2 \cdot 0068\left(400^{\circ} \mathrm{K}\right)$.

Important dopants in YIG are $\mathrm{Gd}^{3+}$ used for reducing $4 \pi M_{\varepsilon}$ and $\mathrm{H}_{0}{ }^{3+}$, $\mathrm{Dy}^{3+}$ used for increasing $h_{\text {crit }}$ and hence power handling capacity. $\mathrm{Gd}^{3+}$ like $\mathrm{Fe}^{3+}$ has a $L=0$ ground state $\left({ }^{3} S_{7 / 2}\right)$ and thus $g=2 \cdot 00$. For Dy ${ }^{3+}, g=1 \cdot 33$ (theor) and $1 \cdot 36$ (exp) while for $H_{0}{ }^{3+}, g=1 \cdot 25$ (theor). $\mathrm{Fe}^{2+}$ which is an undesired presence since it introduces losses due to electron hopping between $\mathrm{Fe}^{2+}$ and $\mathrm{Fe}^{3+}$ has a $g_{\text {eff }}$ value of $1 \cdot 89$. Thus accurate determination of $g_{\text {eff }}$ is important to obtain information on both desired and undesired dopants in YIG.

From the point of device characterisation $g_{\text {err }}$ is directly related to $\gamma$, the gyromagnetic ratio since

$$
\gamma=\left(g e / 4 \pi M_{s}\right) \mathrm{Hz}, \mathrm{Oe} .
$$

For $g=2 \cdot 00$, it is found that $\gamma=2 \cdot 7992 \mathrm{MHz} / \mathrm{Oe}$. For doped crystals $g$ may not be ideally $2 \cdot 00$ and hence the slope of the tuning curve given by (1) may have a different value.

\section{Experimental}

The experimental arrangement, shown in figure 2 , has been previously used by Desormiere (1965) and Masters et al (1960) to measure the narrow line-width of 


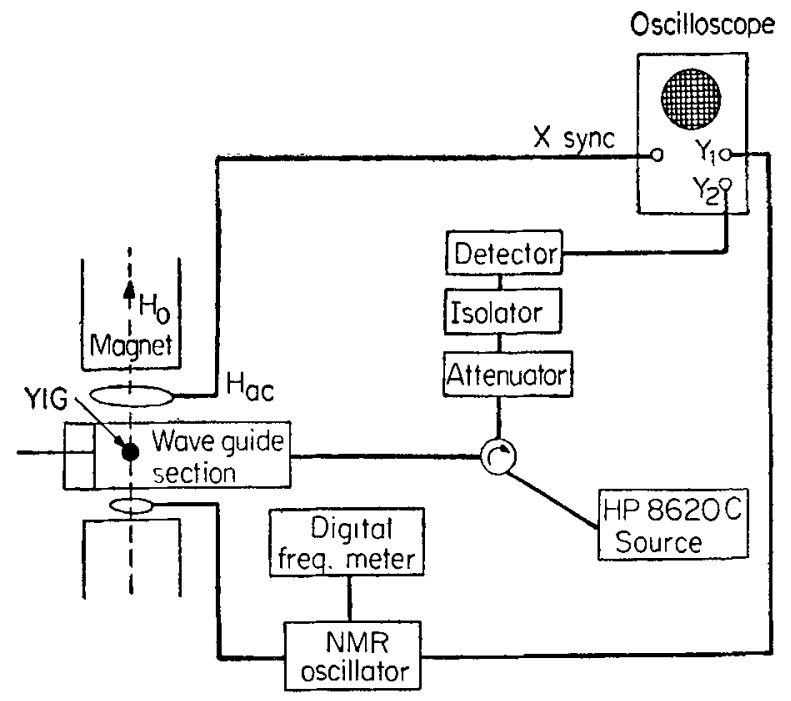

Figure 2. Experimental arrangement for non-resonant measurement of $4 \pi M_{\varepsilon}$ and $g_{\text {eff }}$ of single crystal YIG spheres.

materials such as single crystal YIG. This technique allows variation of spherecircuit interaction by changing the position of the wave-guide short. The YIG sphere is mounted on a perspex rod and is free to rotate and align itself with a $\langle 111\rangle$ easy axis along $H_{0}$. Microwave power is supplied from an HP 8620C source and the reflected power detected and displayed on one channel of an oscilloscope. Power absorbed by the YIG sphere at resonance appears as a dip in the reflected power.

The static magnetic field $H_{0}$ is measured using a proton resonance magnetometer. The ozcillator frequency is measured using a 6-digit frequency meter and its output, with the NMR absorption pip, displayed on another channel of the oscilloscope. A $50 \mathrm{~Hz}$ ac field produced by Helmholtz coils is used to modulate $H_{0}$ and sweep it through resonance. The accuracy of measurement of $H_{0}$ is $\pm 0.1 \mathrm{Oe}$.

For the determization of $4 \pi M_{s} f$ is kept constant and $H_{n}$ varied to first obtain the (110) mode and then the higher order modes, for each of which $H_{0}$ is measured. The mode-chart figure 1 is used for mode-identification.

For the measurement of $g_{\text {eft }}$, the value of $\gamma$ was determined using (1) and $g_{\text {ett }}$ using eq. (8). However since for $\langle 111\rangle / / H_{0}$

$$
H_{\text {eff }}=H_{0}-4 / 3 \quad K_{3} / M_{8}
$$

the anisotropy field $H_{0}=-K_{1} / M_{8}$ need be determined. This was done using the standard method (Matthaei et al 1964) of fixing the sphere and rotating it about a $\langle 110\rangle$ axis and measuring the maximum and minimum $H_{0}$ values for resonance which occur when the $\langle 100\rangle$ and $\langle 111\rangle$ axes respectively lie along $H_{0}$ since:

$$
f=\gamma\left(H_{0}+2 K_{1} / m c\right) \text { for }\langle 100\rangle / / H_{0},
$$

and $f=\gamma\left(H_{0}-4 / 3 K_{1} / M_{\varepsilon}\right)$ for $\langle 111\rangle / / H_{0}$.

Thus for constant $f$,

$$
\left.H_{0}\right|_{100}-\left.H_{0}\right|_{111}=-10 / 3 K_{1} / M_{s} \text {. }
$$


Table 4. Magnetic properties of YIG spheres.

\begin{tabular}{lllllll}
\hline Sphere & $f(\mathrm{GHz})$ & $-\frac{K_{1}}{M_{s}}(\mathrm{Oe})$ & $\mathrm{H}_{\mathrm{eff}}(\mathrm{k} \mathrm{Oe})$ & $\gamma$ & $\mathrm{g}$ & $\triangle \mathrm{H}(\mathrm{Oe})$ \\
\hline \multirow{2}{*}{ WJ-1 } & 10.858 & 43.0 & 3.8788 & 2.7993 & 2.00007 & 0.33 \\
\multirow{2}{*}{ 1-2 } & 10.9 & 43.8 & 3.8864 & 2.8047 & 2.0039 & 0.42 \\
& 11.15 & 43.8 & 3.9777 & 2.8031 & 2.0028 & \\
\multirow{2}{*}{ IGD-1 } & 10.88 & 46.8 & 3.8861 & 2.7997 & 2.0036 & 0.85 \\
& 11.02 & 46.8 & 3.9367 & $\mathbf{2 . 7 9 9 2}$ & 2.0000 & \\
\hline
\end{tabular}

\section{Results}

The results of the first method of determining $4 \pi M_{s}$ using (6) is given in table 2 .

The mode-crossing technique is illustrated by results given in table 3 .

Thus $4 \pi M_{s}=1760 \mathrm{~g}$ for the sphere which was undoped YIG.

A similar procedure used for the $10 \%$ Gd-doped sphere IGD-1 gave $4 \pi M_{s}=$ $1581 \mathrm{~g}$ compared with the values obtained in table 1 and the expected value of $4 \pi M_{s}=1595 \mathrm{~g}$ (Fletcher and Bell 1959).

The results of measurement of $K_{1} / M_{s}$ and hence $g_{\text {eff }}$ are given in table 4 .

\section{Discussion}

The determination of $4 \pi M_{s}$ from higher order magnetostatic modes reported by Fletcher $e t$ al (1959) and used by Lam (1965) was carried out in cavities at a fixed frequency. Large propagation shifts upto $36.6 \mathrm{Oe}$ for the (110) mode, 20 Oe for the (220) mode, etc., occurred, and it was necessary to correct this to obtain agreement between theory and experiment. Electromagnetic propagation effects are neglected in the magnetostatic approximation which assumes $\lambda \gg a$, where $\lambda=$ incident wavelength and $a=$ sphere diameter. Spin-wave propagation is also neglected since it is assumed that $\lambda^{2} \gg H_{\mathrm{ex}} \cdot l / M_{\mathrm{s}}$ where $H_{\mathrm{ex}}=$ equivalent exchange field and $l=$ magnetic lattice constant. Propagaticn shifts have been calculated theoretically by Hurd (1958) for the (110) mode and found experimentally for the other modes. However this theory which makes certain assumptions, like resonance shift being less than $H / 2$, are not valid for low line-width materials.

Magid (1964) employed non-resonant broad band strip-line transmission and reflection structures for measurement of $4 \pi M_{s}$. He used the frequency difference between the (110) and (210) modes which was $700 \mathrm{MHz}$ approximately for undoped YIG. However his measured values were $5-7 \%$ higher than static values for spheres $0.5 \mathrm{~mm}$ dia and as much as $20 \%$ higher for spheres $0.75 \mathrm{~mm}$ dia.

The great advantage of non-resonant waveguide structure is that propagation corrections are not necessary for any modes and the $4 \pi M_{s}$ values are accurately determined by the mode-crossing technique. This is borne out by the measure- 
ment on pure YIG spheres (obtained from Watkins-Johnson (WJ) and prepared at the Indian Institute of Science, Bangalore (I) and also on a Gd-doped sphere. The absence of propagation shifts may be attributed to the use of non-resonant structure because the rf magnetic fields at the sphere are much lower than those in high $Q$ cavities and hence, for a given wavelength and sphere size, the inhomogeneities in the fields are also much less. The interaction between crossing modes was also negligible. However, the fact that the number of higher order modes excited is reduced and the weak modes are not detected acts as a disadvantage. An adequate number of modes are however available for the measurement of $4 \pi M_{B}$ of low line-width spheres.

The validity of the method is also borne out by the accurate values of $g_{\text {eff }}$ obtained without incorporating any propagation corrections. Determination of the frequency of the microwave source which is $\pm 5 \mathrm{MHz}$ gives an error in $g_{\text {eff }}$ of \pm 0.0011 . This can obviously be reduced using a microwave frequency counter. The results may be compared with $g$ values found by Rodrigue et al (1960) which are $g=2.003(a$ site) and 2.0047 ( $d$ site) for pure YIG; $g=1.994 \pm$ 0.005 at $480^{\circ} \mathrm{K}$ for GdIG.

The line-widths of the spheres have also been measured in the same set-up and found to vary between $0.23-0.34$ Oe for the Watkins-Johnson spheres and $0 \cdot 42-0.85$ Oe for spheres grown by us.

\section{Acknowledgements}

The authors are grateful to Prof. A R Vasudeva Murthy and Dr N R Kutty of the Inorganic and Physical Chemistry Department for providing spheres grown in their laboratory.

\section{References}

Artmann J O and Tannenwald P E 1955 J. Appl. Phys. 261124

Desormiere B 1965 Electron. Lett. 2235

Fletcher P C and Bell R O 1959 J. Appl. Phys. 30687

Fletcher P C, Solt I H and Bell R O 1959 Phys. Rev. 114739

Helsajn J 1972 Microwave ferrite engineering (New York: Wiley Interscience)

Hurd R A 1958 Can. J. Phys. 361072

Lam Y W 1965 Solid State Electron. 8923

Magid M 1964 IEEE Trans. IM-13 329

Masters J I, Capone B R and Gianino P D 1960 IEEE Trans. MTT-8 565

Matthaei G L, Young L and Jones E M T 1964 Microwave filters, impedance matching networks and coupling structures (New York: McGraw-Hill)

Narayan K G, Sharma G P and Pran Kishan 1969 IEEE Trans. IM-18 114

Rodrigue G P, Meyer A and Jones R V 1960 J. Appl. Phys. 31376

von Aulock H 1965 Handbook of Microwave Ferrite materials (London: Academic Press) 